\title{
Recovery from Posttraumatic Stress Requires Dynamic and Sequential Shifts in Amygdalar Connectivities
}

\author{
Sujung Yoon ${ }^{1,2,8}$, Jieun E Kim ${ }^{1,2,8}$, Jaeuk Hwang ${ }^{3}$, Ilhyang Kang ${ }^{1,2}$, Saerom Jeon ${ }^{1,2}$, Jooyeon J Im ${ }^{1,4}$, \\ Bori R Kim ${ }^{1,2}$, Sunho Lee,4, Geon Ha Kim', Hyewhon Rhim ${ }^{5}$, Soo Mee Lim ${ }^{6}$ and In Kyoon Lyoo*,1,2,7 \\ 'Ewha Brain Institute, Ewha Womans University, Seoul, South Korea; '2Department of Brain and Cognitive Sciences, Ewha Womans University, \\ Seoul, South Korea; ${ }^{3}$ Department of Psychiatry, Soonchunhyang University College of Medicine, Seoul, South Korea; ${ }^{4}$ Interdisciplinary Program in \\ Neuroscience, College of Natural Sciences, Seoul National University, Seoul, South Korea; ${ }^{5}$ Center for Neuroscience, Korea Institute of Science and \\ Technology, Seoul, South Korea; ${ }^{6}$ Department of Radiology, Ewha Womans University College of Medicine, Seoul, South Korea; ${ }^{7}$ College of \\ Pharmacy, Graduate School of Pharmaceutical Sciences, Ewha Womans University, Seoul, South Korea
}

\begin{abstract}
The neural mechanisms underlying the development and maintenance of posttraumatic stress disorder (PTSD) have long been studied. However, little is known about the neural correlates of the recovery process from PTSD. A 5-year longitudinal study was conducted to investigate the trajectory of structural connectivities of the amygdala in disaster survivors with PTSD. Thirty disaster survivors, who were diagnosed with PTSD, and 29 healthy individuals, who were not exposed to trauma, underwent three waves of assessments including neuroimaging scanning over a 5-year period from the time of the disaster at approximately 1.3-year intervals. All disaster survivors showed significant improvements in PTSD symptoms over time. Using diffusion tensor imaging analysis, a 5-year trajectory of amygdalar structural connectivities with key brain regions was assessed. The amygdala-insula connection was initially strengthened and then normalized during recovery, while the amygdala-prefrontal cortex (PFC) connection was at first unaffected, then strengthened, and eventually normalized. The lower tract strength of the amygdala-thalamus connection normalized during recovery, while that of amygdala-hippocampus connection remained low. The greater amygdala-PFC connectivity was associated with less PTSD symptom severity. The present longitudinal study revealed that recovery from PTSD parallels dynamic and sequential shifts in amygdalar connectivities with multiple brain regions, suggesting the expanded view of fear circuitry including the insula and thalamus, beyond the traditional model which primarily involves the amygdala, PFC, and hippocampus.

Neuropsychopharmacology (2017) 42, 454-46I; doi:I0.1038/npp.2016.136; published online 17 August 2016
\end{abstract}

\section{INTRODUCTION}

Learning about environmental threats is an evolutionarily adaptive function in humans and just as critical is adjusting learned fear in restitution of an emotionally neutral state. Maladaptive processing of fear memory can entail excessive anxiety (Shin and Liberzon, 2010; Pitman et al, 2012; Giustino and Maren, 2015; Admon et al, 2013; Yehuda and LeDoux, 2007), which may lead to the development of anxiety disorders, such as posttraumatic stress disorder (PTSD).

Hyperresponsivity of the amygdala, decreased top-down control by the prefrontal cortex (PFC), and dysfunction of the hippocampus in the contextualization of threats and safety signals have been reported to be mainstays for sustained fear memory and key pathophysiology of PTSD (Giustino and

*Correspondence: Professor IK Lyoo, Ewha Brain Institute and Department of Brain and Cognitive Sciences, Ewha Womans University, 52 Ewhayeodae-gil, Seodaemun-gu, Seoul 03760, South Korea, Tel: +82 23277 6550, Fax: +82 232776562 ,

E-mail: inkylyoo@ewha.ac.kr

${ }^{8}$ These authors contributed equally to this work.

Received 17 May 2016; revised II July 2016; accepted 19 July 2016; accepted article preview online 27 July 2016
Maren, 2015; Admon et al, 2013; Milad et al, 2007; Pitman et al, 2012). Despite growing knowledge of the neural basis for the acquisition, maintenance, and extinction of fear memory that primarily involves the amygdala-centered circuit, including the PFC and hippocampus, not much is known about the neural correlates of recovery from PTSD in humans.

Moreover, it has not been identified whether recovery from PTSD takes a timed sequence of brain reorganization involving multiple brain regions or is rather straightforward reversal/restoration of PTSD-related brain abnormalities. Consequently, we intended to longitudinally assess the white matter tract strength between the amygdala and its connected brain regions of the fear circuitry during the recovery process from PTSD.

Emerging evidence has suggested that other brain areas beyond the traditional fear circuitry, such as the insula and thalamus, are involved in the pathophysiology of PTSD (Koch et al, 2016; Patel et al, 2012; Paulus and Stein, 2006; Pitman et al, 2012). Therefore, we have broadened our scope to include the thalamus and insula, which also have connections with the amygdala.

In this multiwave study, 30 disaster survivors who were diagnosed with PTSD in the early aftermath of the trauma 
and 29 healthy individuals who were not exposed to trauma were prospectively followed for up to 5 years as the traumaexposed and trauma-unexposed groups.

\section{MATERIALS AND METHODS}

\section{Participants}

Study participants were recruited from a group of survivors of a subway fire disaster in South Korea (Lyoo et al, 2011). Thirty-eight individuals participated in time 0 assessment for the diagnosis and the symptom severity of PTSD at approximately 1.65 months after the disaster. Thirty traumaexposed individuals who participated in the following multiwave assessments of brain magnetic resonance imaging (MRI) scans and comprehensive psychiatric evaluations were eventually included in the final analysis. Among 36 controls of the original cohort (Lyoo et al, 2011), 29 age- and sexmatched individuals who undertook two or more assessments were included in the trauma-unexposed group. Detailed information on the inclusion and exclusion criteria for study participation is described in Supplementary Information.

Baseline (time 0) and three serial assessments (times 1-3) were performed over a 5-year period from the time of the disaster at 1.65 months (time 0), 1.43 years (time 1), 2.68 years (time 2), and 3.91 years (time 3 ) since trauma with approximately 1.3-year intervals. Among 30 trauma-exposed individuals who underwent time 1 assessment, 25 were evaluated at time 2 assessment and 17 completed all three waves of assessments. In the trauma-unexposed group, 29 individuals completed times 1 and 2 assessments and 21 were evaluated at time 3 assessment. Time points of assessments and the number of participants at each time point are shown in Supplementary Figure S1.

Assessment of PTSD symptom severity and diagnosis of PTSD were performed using the Clinician-Administered Posttraumatic Stress Disorder Scale (CAPS; Blake et al, 1995) and the Structural Clinical Interview for the DSM-IV (First et al, 1996), respectively. Attainment and maintenance of reliability for clinical measurements over the study period is described in our previous report (Lyoo et al, 2011).

This study was approved by the Institutional Review Board of the Seoul National University Hospital.

\section{Neuroimaging Analysis}

Three waves of serial brain MRI scans were performed using a 3-Tesla whole-body imaging system (Signa EXCITE; GE Medical Systems, Milwaukee, WI). High-resolution T1weighted and diffusion tensor brain MR images were obtained.

Diffusion tensor imaging processing. Diffusion tensor imaging (DTI) data were processed using the FMRIB Software (FSL; http://www.fmrib.ox.ac.uk/fsl). In order to correct for head motion and minimize distortions caused by eddy current, diffusion-weighted images were registered to the non-diffusion image $\left(b=0 \mathrm{~s} / \mathrm{m}^{2}\right)$ by affine transformations using the FMRIB's Linear Image Registration Tool implemented in the FMRIB's Diffusion Toolbox.

Amygdalar seed and the target brain regions. Seed and target regions for fiber tracking included the regions of the amygdala, which was derived from the manually segmented label (Supplementary Materials and Methods), and the orbitofrontal and adjacent ventromedial prefrontal cortex (OMPFC), hippocampus, insula, and thalamus (Saygin et al, 2011), which were derived from the standard automated anatomical labeling (AAL) map (Tzourio-Mazoyer et al, 2002), respectively (Supplementary Figure S2). The OMPFC region consisted of the ventromedial prefrontal cortex and the orbitofrontal cortex (Ongur and Price, 2000; Gusnard et al, 2003). Interhemispheric fibers and tracts originating from or terminating in other cortical regions were restricted using the relevant exclusion masks of the contralateral hemisphere and the ipsilateral occipital and parietal cortices. Registration steps for seed and target brain regions are described in Supplementary Materials and Methods.

Seed-based classification and the measurement of amygdalar connectivities. Probabilistic tractography, which modeled probability distributions on two fiber directions at each voxel level, was performed using the FSL's bedpostX program based on a multifiber diffusion model, which may account for the issue related to crossing fibers (Behrens et al, 2007). Probabilistic fiber tracking from the amygdala (seed region) to the target brain regions of the OMPFC, hippocampus, insula, and thalamus was carried out with 5000 tract-following samples at each amygdalar seed voxel, separately for each hemisphere, with a curvature threshold of 0.2. All probabilistic tractography was performed in each individual's diffusion space. Visual inspection of all resultant tract images was performed to ensure the acceptability for further analyses.

The relative tract strength, which were calculated using a classification procedure, were the measures for amygdalar connectivities with the OMPFC, hippocampus, insula, and thalamus. Details regarding seed-based classification for measuring the relative tract strength and exploratory voxelwise analyses of the tract strength values are presented in Supplementary Materials and Methods.

\section{Statistical Analyses}

Independent $t$-tests and chi-square tests were, respectively, used to compare continuous and categorical demographic or psychometric variables between groups.

Using the group means and SDs of amygdalar volume and tract strength in the trauma-unexposed (control) group as the reference values, adjusted amygdalar volume and tract strength were converted into standardized $Z$-scores and used in the final analysis. A negative $Z$-score indicates that adjusted amygdalar volume or tract strength was below the mean of the trauma-unexposed group in SD units.

The general linear model was used for the group comparisons of standardized $Z$-scores of amygdalar volume at each time point. Multivariate multiple regression analysis was used for the group comparisons of standardized $Z$-scores of the mean tract strength at each time point. To account for multiple comparisons, the permutation-adjusted $p$-values for each measure were calculated (Westfall and Young, 1993). A total of 5000 permutations for the group status (traumaexposed $v s$ trauma-unexposed groups) were performed. The proportion of permutations with a permuted $p$-value of the null distribution that was greater than the original $p$ - 
Table I Demographic and Clinical Characteristics of Study Participants

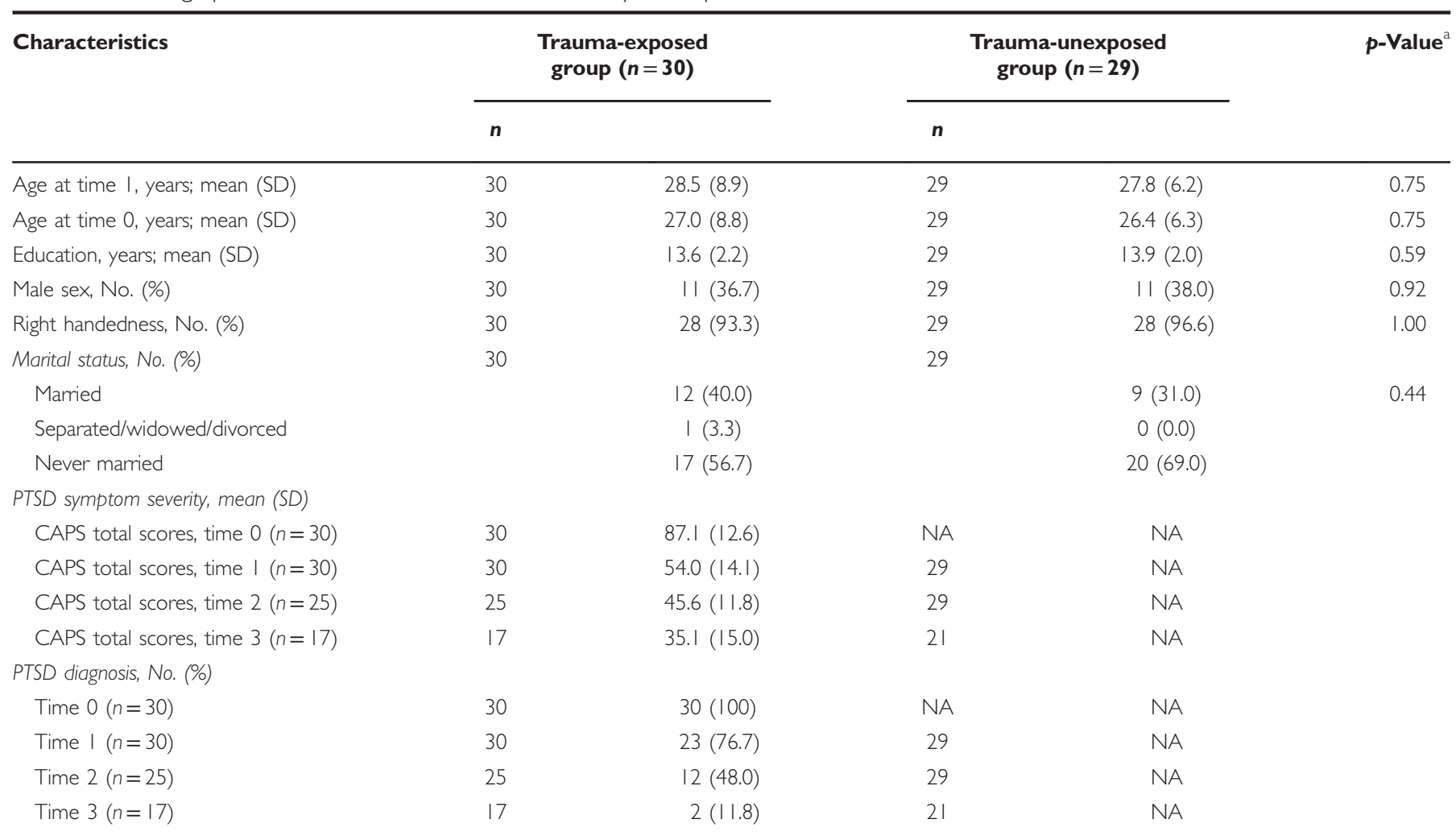

Abbreviations: CAPS, Clinician-Administered Posttraumatic Stress Disorder Scale; PTSD, posttraumatic stress disorder.

${ }^{a} p$-Values were computed by $\chi^{2}$ or Fisher's exact tests for categorical measures and independent $t$-tests for continuous measures.

value from the actual data set was computed as a permutation-adjusted $p$-value (Westfall and Young, 1993).

Changes in the standardized $Z$-scores of the mean tract strength were assessed in each trauma-exposed and traumaunexposed group using the generalized estimating equation (GEE), which can minimize the effects of missing data (Hennen, 2003). A linear time effect was the predictor of interest. The linear and quadratic models for time effects on the mean tract strength were also constructed and compared using the likelihood ratio test to select the bestfitting model.

The GEE, which allows for the within-subject correlation, was also used to examine the relationships between the standardized $Z$-scores of the mean tract strength and PTSD symptom severity.

Two-tailed significance at $p<0.05$ was considered as statistically significant. Data were analyzed using the Stata SE, v11.0 (Stata, College Station, TX).

\section{RESULTS}

There were no significant differences in demographic and clinical characteristics between trauma-exposed and traumaunexposed individuals at each time point and between individuals who completed all three assessments and those who did not. Detailed information is described in Table 1 and Supplementary Information.
All trauma-exposed individuals showed significant improvements in PTSD symptoms over time (linear time effect, $z=-16.0, p<0.001)$, as measured using the CAPS (Supplementary Figure S3A). Among trauma-exposed individuals all of whom had PTSD at time 0,7 of $30(23 \%)$ who were assessed at time 1,13 of $25(52 \%)$ at time 2, and 15 of $17(88 \%)$ at time 3 improved substantially and did not meet the diagnostic criteria for PTSD (Table 1).

\section{Tract Strength Differences at Each Time Point}

At time 1 (a mean of 1.4 years after the trauma) when $77 \%$ of the individuals $(n=23)$ still met the diagnostic criteria for PTSD, the trauma-exposed group had greater tract strength between the amygdala and insula relative to the traumaunexposed group $(b=0.67$, permutation-adjusted $p=0.02)$. In contrast, the tract strength of the amygdalar connections with the thalamus $(b=-0.62$, permutation-adjusted $p=0.02)$ and with the hippocampus $(b=-0.54$, permutation-adjusted $p=0.04$ ) were lower in the trauma-exposed group relative to the trauma-unexposed group. There was no significant difference in the amygdala-OMPFC tract strength between the trauma-exposed and trauma-unexposed groups $(b=$ -0.20 , permutation-adjusted $p=0.48$; Figure 1 ).

At time 2 (a mean of 2.7 years after the trauma), $48 \%$ $(n=12)$ of the trauma-exposed individuals who were assessed at that time $(n=25)$ met the diagnostic criteria for PTSD. Interestingly, the amygdala-OMPFC tract strength was greater in the trauma-exposed group than in the trauma- 


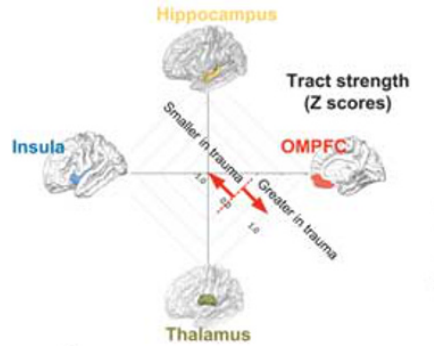

$Z$ scores of the trauma-exposed group

$Z$ scores of the control group

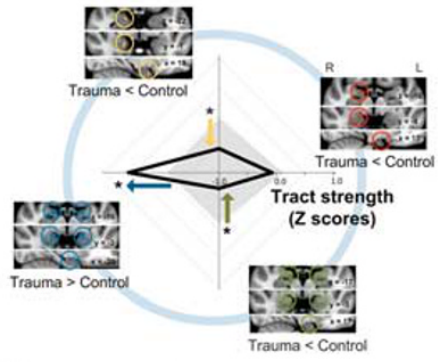

Time 1

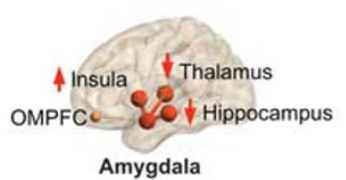

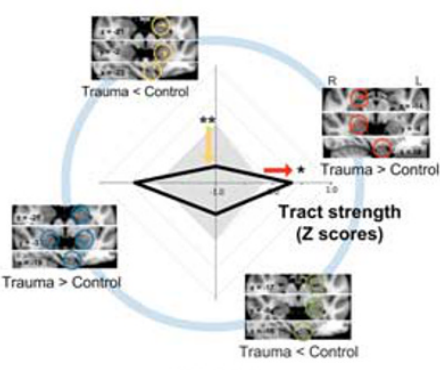

Time 2

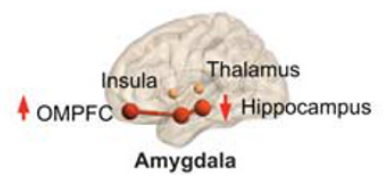

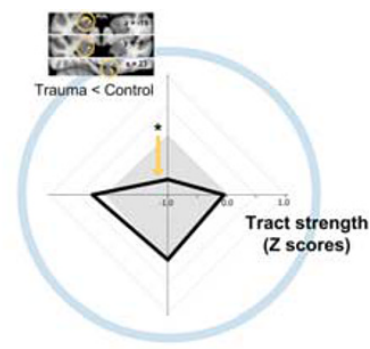

Time 3

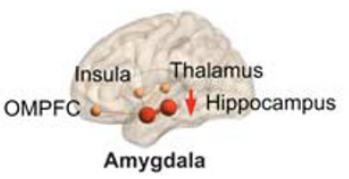

Figure I Comparisons of the tract strength connecting the amygdala to the OMPFC (right, red), thalamus (lower, green), insula (left, blue), and hippocampus (upper, yellow) between the trauma-exposed and trauma-unexposed groups at each time point. Standardized Z-scores of the tract strength in the radar charts were calculated using the mean and SD of trauma-unexposed individuals after adjusting for age and sex. Radar charts at each time point represent comparisons in the tract strength between the trauma-exposed (black solid lines) and the trauma-unexposed (gray solid lozenges) groups. Multivariate multiple regression analysis was used to compare the standardized $Z$-scores of tract strength between groups at each time point. An exploratory voxel-wise analysis was also performed to find clusters of significant group effects on the relative tract strength values at corrected $p<0.05$ (see also Supplementary Materials and Methods and Supplementary Table SI). Relevant clusters are overlaid on the standard MNI template. 'Trauma $<$ Control' indicates significant clusters of smaller tract strength in the trauma-exposed than in the trauma-unexposed groups, while 'Trauma $>$ Control' indicates those of greater tract strength in the trauma-exposed than in the trauma-unexposed groups. *Permutation-adjusted $p<0.05$ (5000 permutations) and *** adjusted $p<0.01$ (5000 permutations).
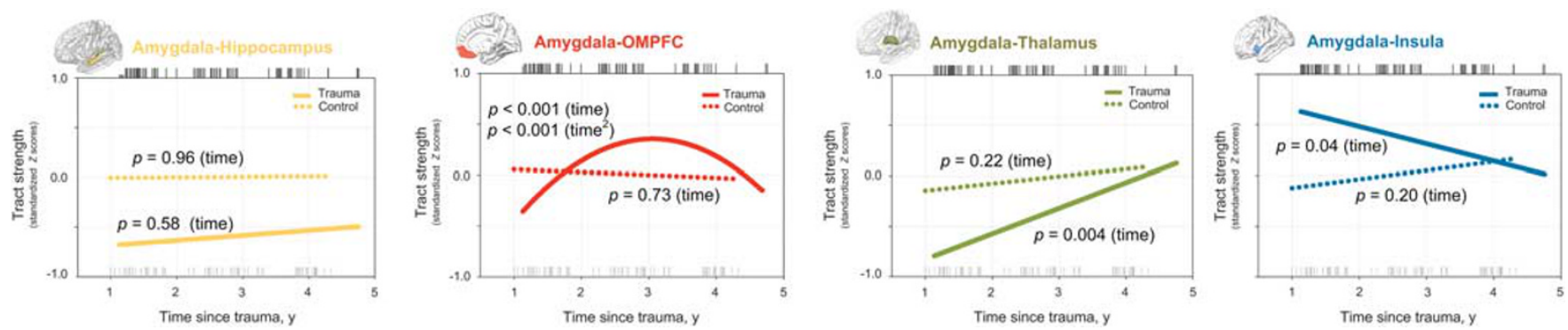

Figure 2 Estimated changes in the tract strength connecting the amygdala to the hippocampus (yellow), OMPFC (red), thalamus (green), and insula (blue) for the trauma-exposed (solid lines) and control (dotted lines) groups. Regression lines for changes in the tract strength over time were fitted using the generalized estimating equation model. Black and gray tick marks above and below the fitted lines in the graphs indicate times since trauma at each assessment in the trauma-exposed and trauma-unexposed groups, respectively. p-Values in the graphs are with regard to the significance of the linear (time) or quadratic $\left(\right.$ time $\left.^{2}\right)$ time effects on the tract strength.

unexposed group at time $2(b=0.47$, permutation-adjusted $p=0.04$; Figure 1).

At time 3 (a mean of 3.9 years after the trauma) when only 2 of the 17 trauma-exposed individuals met diagnostic criteria for PTSD, the increased tract strength between the amygdala and OMPFC at time 2 had decreased to a normal level similar to that of the trauma-unexposed group $(b=-0.08$, permutation-adjusted $p=0.80$; Figure 1 ).

Although the tract strength between the amygdala and hippocampus in the trauma-exposed group remained low (time 2, $b=-0.74$, permutation-adjusted $p=0.009$; time 3 , $b=-0.76$, permutation-adjusted $p<0.05$ ), those between the amygdala and insula/thalamus normalized over time (time 2: insula, $b=0.38$, permutation-adjusted $p=0.20$; thalamus, $b=-0.45$, permutation-adjusted $p=0.13$; and time 3 : insula, $b=0.20$, permutation-adjusted $p=0.58$; thalamus, $b=-0.04$, permutation-adjusted $p=0.89$; Figure 1 ).

The results were similar when the analyses were repeated in the subsample of participants who completed all three assessments (17 trauma-exposed and 21 trauma-unexposed individuals; Supplementary Result S1) or when considering a potential confounding factor including the experience of sub-PTSD-level lifetime stressful events (Supplementary Result S2).

\section{Tract Strength Changes Over Time in Each Group}

In the trauma-exposed group, changes in the tract strength between the amygdala and OMPFC from time 1 through time 3 were fitted with linear $(z=3.75, p<0.001)$ and quadratic $(z=-3.64, p<0.001)$ time terms that were characterized by a pattern of an earlier increase and a later decrease to a normal level (Figure 2). The tract strength between the amygdala and thalamus increased $(z=2.88$, $p=0.004)$ while that between the amygdala and insula decreased over time $(z=-2.09, p=0.04)$ throughout the study period in the trauma-exposed group. In contrast, the amygdala-hippocampus tract strength did not change over 

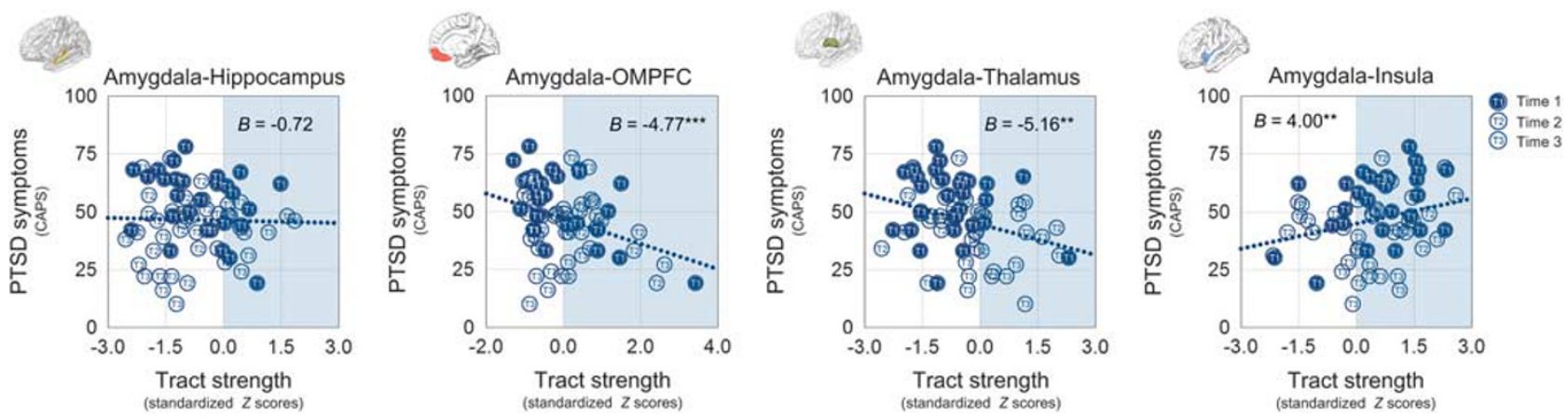

Figure 3 Relationships between amygdalar tract strength to each brain region and PTSD symptom severity. Scatter plots and regression lines indicate the relationships between the amygdalar tract strength to each target region and PTSD symptom severity. Values in blue shaded areas in each scatter plot indicate positive standardized $Z$-scores representing values above the means of the trauma-unexposed group in SD units. $* * p<0.01$ and $* * * p<0.001$.

time in the trauma-exposed group $(z=0.56, p=0.58$; Figure 2).

There were no changes in the amygdalar tract strength to the hippocampus $(z=0.06, p=0.96)$, OMPFC $(z=-0.35$, $p=0.73)$, thalamus $(z=1.21, p=0.22)$, and insula $(z=1.27$, $p=0.20$ ) over time in the trauma-unexposed group (Figure 2).

The results of repeated analyses of participants who completed all three assessments or when considering potential confounding factors, including comorbid depression and the experience of stressful events, remained similar ensuring the robustness of the current findings (Supplementary Results S1 and S2).

\section{Relationship Between Amygdalar Tract Strength and PTSD Symptom Severity}

The greater amygdala-OMPFC tract strength was associated with less severe PTSD symptoms $(z=-3.87, p<0.001)$. PTSD symptom severity was positively associated with the amygdala-insula tract strength $(z=2.80, p=0.005)$ and negatively with the amygdala-thalamus tract strength $(z=$ $-3.26, p=0.001)$, respectively. In contrast, the amygdalahippocampus tract strength was not associated with PTSD symptom severity $(z=-0.38, p=0.70 ;$ Figure 3$)$. The relationships between changes in amygdalar connectivity and PTSD symptom improvement were also explored and presented in Supplementary Result S3.

\section{Amygdalar Volume Differences at Each Time Point}

We found that amygdalar volumes were smaller in the trauma-exposed group than in the trauma-unexposed group at time 1 (left, $b=-0.59$, permutation-adjusted $p=0.02$; right, $b=-0.47$, permutation-adjusted $p=0.07$; total, $b=$ -0.57 , permutation-adjusted $p=0.02$; Supplementary Figure S3B). There were no differences in amygdalar volumes between the trauma-exposed and trauma-unexposed groups at time 2 (left, $b=-0.23$, permutation-adjusted $p=0.35$; right, $b=-0.35$, permutation-adjusted $p=0.18$; total, $b=-0.33$, permutation-adjusted $p=0.20$ ) and at time 3 (left, $b=-0.03$, permutation-adjusted $p=0.93$; right, $b=-0.05$, permutation-adjusted $p=0.86$; total, $b=-0.05$, permutationadjusted $p=0.88$; Supplementary Figure S3B). Time effects on amygdalar volumes were also explored in each group and presented in Supplementary Result S4.

\section{DISCUSSION}

To the best of our knowledge, the current longitudinal study on a survivor cohort with PTSD provides the first brain structural trajectory of the amygdala and key brain regions that are connected to the amygdala over a 5-year recovery period following trauma exposure. The present study included disaster survivors, who initially met the diagnostic criteria for PTSD and had substantial improvements in PTSD symptoms over a 5-year period. Considering that most previous studies have examined the neural correlates of current PTSD, this longitudinal multimodal neuroimaging study provides a unique perspective on the brain processes involved in recovery from PTSD.

The heightened connectivity between the amygdala and insula was a notable finding at time 1 , when most of the trauma-exposed individuals had clinically significant PTSD symptoms. Furthermore, greater amygdalar connectivity with the insula was associated with more severe PTSD symptoms. Recently, there has been increasing interest in the potential role of the insula in mediating fear-related anxiety. Individuals with a dysfunctional insula may misinterpret the effects of environmental stimuli on the body and then consider normal interoceptive state dangerous (Patel et al, 2012; Paulus and Stein, 2006). Strengthened connections between the amygdala and insula at time 1 thus may render trauma-exposed individuals to become hypersensitive to internal body signals as well as to external environmental stimuli. This may prompt hyperarousal symptoms and overt physiological manifestations of PTSD (Sripada et al, 2012a). The decreased strength of thalamic connections with the amygdala at time 1 may also contribute to the dysfunctional action of the insula on exaggerated interpretation of the bodily state, by reducing transfer of sensory information (Lanius et al, 2006; Patel et al, 2012). This assumption was corroborated by the significant relationships between amygdalar connectivities with the insula or thalamus and PTSD symptom severity found in this study.

Our findings are also consistent with the recent functional connectivity studies of individuals with PTSD (Rabinak et al, 2011; Sripada et al, 2012a; Sripada et al, 2012b), given that brain regions that are linked via white matter tracts are also strongly functionally connected (Damoiseaux and Greicius, 2009; Honey et al, 2010). Increased functional coupling between the amygdala and insula, which can mediate anxious 
and emotional anticipation of aversive stimuli (Carlson et al, 2011) and then potentially evoke PTSD symptoms, has been observed in individuals with current PTSD (Rabinak et al, 2011; Sripada et al, 2012a; Sripada et al, 2012b).

Although the pathophysiological role of connectivity changes between the amygdala and thalamus in PTSD has not yet been actively explored, it has been reported that individuals with current PTSD showed decreased activation in the thalamus (Kim et al, 2007; Lanius et al, 2001). In addition, altered functional connectivity between the PFC and thalamus (Yin et al, 2011), implying the dysfunctional prefrontal control over the sensory gating of the thalamus, has also been observed in PTSD patients. Our findings of the lower amygdalar connectivity with the thalamus, which characterized the trauma-exposed group at time 1, may underlie the disrupted relay of sensory information to the amygdala (Lanius et al, 2006) and in turn resulted in increasing PTSD symptom severity.

Importantly, our data show that the dynamic changes in the connection between the amygdala and OMPFC are closely related to recovery from PTSD. The role of the OMPFC in fear processing is not limited to the extinction of fear memory (Schiller and Delgado, 2010; Yehuda and LeDoux, 2007). Ongoing modulation including reversal and cognitive re-evaluation of fear memory is known to require the involvement of the OMPFC (Quirk and Beer, 2006; Schiller and Delgado, 2010). The heightened amygdalaOMPFC connectivity at time 2 in the trauma-exposed group may imply the successful inhibitory control of the OMPFC over the amygdalar hyperactivity at this stage. In addition, the greater tract strength between the amygdala and OMPFC was associated with less severe PTSD symptoms. This is in line with a recent functional connectivity study on PTSD that suggested negative correlation between the PFC-amygdala connection and symptom severity (Brown et al, 2014).

In a previous study using the same cohort, the dorsolateral prefrontal cortex (DLPFC) was first reported to have an important role in fear modulation during recovery (Lyoo et al, 2011). As the DLPFC and amygdala do not share direct anatomical connections, the OMPFC could be potentially mediating this modulation (Quirk and Beer, 2006; Schiller and Delgado, 2010). As an exploratory analysis, we examined the potential role of the DLPFC in controlling the fear circuitry (Depue et al, 2007) through its connections with the OMPFC (Supplementary Result S5 and Supplementary Figure S4). Interestingly, changes in white matter integrity of the DLPFC-OMPFC tract were fitted with linear $(z=3.12$, $p=0.002)$ and quadratic $(z=-2.47, p=0.01)$ time terms in the trauma-exposed group. However, white matter integrity in these intralobar frontal connections did not change over time in the trauma-unexposed group. Our exploratory analysis shows that increased white matter integrity between the DLPFC and OMPFC at time 2 may follow the cortical thickness increase in the DLPFC at time 1 in the traumaexposed group (Lyoo et al, 2011). This implies that a close association among the DLPFC, OMPFC, and amygdala may importantly contribute to recovery from PTSD. However, these preliminary results should be replicated in a larger sample.

It is intriguing that the increase in white matter tract strength between the OMPFC and amygdala appears to be accompanied by the normalizing pattern in the connections between the amygdala and insula/thalamus. This increase in connection strength between the OMPFC and amygdala at time 2 subsequently normalized at time 3 .

The amygdala-hippocampus connectivity did not change and remained low in trauma-exposed individuals even after substantial recovery from PTSD had been achieved. This finding may raise an issue of preexistence $v s$ ongoing adaptation regarding the origins of hippocampal pathology in PTSD (Yehuda and LeDoux, 2007). Given that the hippocampus has a critical role in context-dependent extinction of fear memory (Schiller and Delgado, 2010), low amygdala-hippocampus connectivity may also have led our disaster survivors to develop PTSD or early fear responses. However, it is also noted that the amygdalahippocampus connectivity was assessed at time 1, a mean of 1.4 years after the trauma. Therefore, the possibility of the amygdala-hippocampus connectivity reduction in response to trauma exposure should be considered.

At time 1, smaller amygdalar volumes were observed in the trauma-exposed group than in the trauma-unexposed group. Although previous cross-sectional studies have reported inconsistent results with regard to PTSD-related amygdalar volume abnormalities (Kuo et al, 2012; Morey et al, 2012; Woon and Hedges, 2009), smaller and hyperactive amygdala in response to a traumatic event has been suggested to constitute a risk for the development of PTSD (Admon et al, 2009). However, little is known about the reversibility of this pathology with symptom improvements. Given that the amygdalar volume was smaller in the trauma-exposed group than in the trauma-unexposed group at time 1, but not at times 2 and 3 when PTSD symptoms substantially improved, our results imply the trend toward normalization of the amygdalar volume throughout recovery from PTSD. However, as we did not find the significant time effects on the amygdalar volume in the trauma-exposed group potentially owing to a small sample size, future studies with a larger sample size will be needed.

In contrast to PTSD samples studied in most previous neuroimaging studies (Hedges, 2007; Karl et al, 2006), most of the survivors in this longitudinal cohort experienced a single traumatic event and recovered during the 5 years after trauma (Lyoo et al, 2011). In addition, the interval between trauma exposure and neuroimaging taken seems to be shorter in this study (the first-wave assessment approximately 1 year after trauma) than those in previous studies (mostly several years after trauma). The longitudinal observation during recovery from PTSD after a single discrete trauma may provide important information regarding the brain trajectories of recovery from PTSD. However, to make the results more generalizable, longitudinal brain trajectories of chronic unremitting PTSD after repeated traumatic experiences needs to be studied in the future research with a larger heterogeneous sample.

Considering a high attrition rate that has commonly been reported in cohort studies of disaster survivors (Galea et al, 2005), it is important to take this into account in interpreting the results. We used an appropriate statistical method to minimize the effects of missing data (Hennen, 2003). In addition, similar results from repeated analyses including study participants who completed all three assessments (Supplementary Result S1) imply that current findings may not be attributed to the high dropout rates. 
Individuals who had experienced the traumatic event but did not develop PTSD (trauma-exposed non-PTSD control group) were not included in this study. Therefore, the current study design could not discriminate the brain regional alterations related to trauma exposure from those associated with PTSD. Given that recovery from PTSD may involve changes in pathogenic processes, including brain responses to both trauma exposure and PTSD, the current findings may represent trajectories of amygdalar connectivity changes during recovery from both pathogenic processes. The recent systemic reviews have also suggested that the comparison between the trauma-exposed and trauma-unexposed control groups may provide information on combined brain regional alterations related to trauma exposure and disease ( $\mathrm{Li}$ et al, 2014; Patel et al, 2012).

Given a relatively small sample size of the cohort, the current longitudinal findings should be replicated in a larger cohort, including trauma-exposed individuals who have recovered from PTSD and those who have not developed PTSD.

Although there is no clear evidence available to aid in the selection of appropriate time interval to examine changes in amygdalar structural connectivities, more frequent acquisition of clinical and neuroimaging data would have provided valuable information regarding the earlier brain structural changes related to recovery from PTSD.

A limitation of the current study is that functional or behavioral tasks, which may link changes in amygdalar connectivity to specific brain functions related to fear responses, were not included. Future studies with specific behavioral tasks will likely provide the functional significance of dynamic changes in amygdalar connectivity in traumaexposed individuals.

In the current study, we have shown a 5-year trajectory of amygdalar connectivities with key brain regions during recovery from PTSD. An expanded brain network, including the insula, thalamus, and DLPFC, which extends beyond the traditional fear circuitry of the amygdala, OMPFC, and hippocampus, appears to be involved in recovery from PTSD.

\section{FUNDING AND DISCLOSURE}

This project was supported by 2015M3C7A1028373, 2015M3C7A1028376, and 2013R1A2A2A01010309 grants from the National Research Foundation of Korea. The funders of the study had no role in the design and conduct of the study; in the collection, analysis, and interpretation of the data; in the preparation, review, or approval of the manuscript; or in the decision to submit the manuscript for publication. The authors declare no conflict of interest.

\section{ACKNOWLEDGMENTS}

We thank the invaluable contributions of the participants of this 5-year longitudinal follow-up study. We also thank Sandy Jeong Rhie for English language editing of the manuscript. An abstract of the preliminary data from this study was presented as a poster at the Brain Conference, the Korean Society for Brain and Neural Science 2014 Annual Meeting on 6 November 2014.

\section{REFERENCES}

Admon R, Lubin G, Stern O, Rosenberg K, Sela L, Ben-Ami H et al (2009). Human vulnerability to stress depends on amygdala's predisposition and hippocampal plasticity. Proc Natl Acad Sci USA 106: 14120-14125.

Admon R, Milad MR, Hendler T (2013). A causal model of post-traumatic stress disorder: disentangling predisposed from acquired neural abnormalities. Trends Cogn Sci 17: 337-347.

Behrens TE, Berg HJ, Jbabdi S, Rushworth MF, Woolrich MW (2007). Probabilistic diffusion tractography with multiple fibre orientations: what can we gain? Neuroimage 34: 144-155.

Blake DD, Weathers FW, Nagy LM, Kaloupek DG, Gusman FD, Charney DS et al (1995). The development of a ClinicianAdministered PTSD Scale. J Trauma Stress 8: 75-90.

Brown VM, LaBar KS, Haswell CC, Gold AL, McCarthy G, Morey RA (2014). Altered resting-state functional connectivity of basolateral and centromedial amygdala complexes in posttraumatic stress disorder. Neuropsychopharmacology 39: 351-359.

Carlson JM, Greenberg T, Rubin D, Mujica-Parodi LR (2011). Feeling anxious: anticipatory amygdalo-insular response predicts the feeling of anxious anticipation. Soc Cogn Affect Neurosci 6: 74-81.

Damoiseaux JS, Greicius MD (2009). Greater than the sum of its parts: a review of studies combining structural connectivity and restingstate functional connectivity. Brain Struct Funct 213: 525-533.

Depue BE, Curran T, Banich MT (2007). Prefrontal regions orchestrate suppression of emotional memories via a two-phase process. Science 317: 215-219.

First MB, Spitzer RL, Gibbon M., Williams JB. (1996). Structured Clinical Interview for DSM-IV Axis I Disorders (SCID-II), Version 2. Biometrics Research, New York State Psychiatric Institute: New York, USA.

Galea S, Nandi A, Vlahov D (2005). The epidemiology of post-traumatic stress disorder after disasters. Epidemiol Rev 27: 78-91.

Giustino TF, Maren S (2015). The role of the medial prefrontal cortex in the conditioning and extinction of fear. Front Behav Neurosci 9: 298.

Gusnard DA, Ollinger JM, Shulman GL, Cloninger CR, Price JL, Van Essen DC et al (2003). Persistence and brain circuitry. Proc Natl Acad Sci USA 100: 3479-3484.

Hedges DW (2007). Structural magnetic resonance imaging findings in posttraumatic stress disorder and their response to treatment. Curr Psychiatry Rev 3: 85-93.

Hennen J (2003). Statistical methods for longitudinal research on bipolar disorders. Bipolar Disord 5: 156-168.

Honey CJ, Thivierge JP, Sporns O (2010). Can structure predict function in the human brain? Neuroimage 52: 766-776.

Karl A, Schaefer M, Malta LS, Dorfel D, Rohleder N, Werner A (2006). A meta-analysis of structural brain abnormalities in PTSD. Neurosci Biobehav Rev 30: 1004-1031.

Kim SJ, Lyoo IK, Lee YS, Kim J, Sim ME, Bae SJ et al (2007). Decreased cerebral blood flow of thalamus in PTSD patients as a strategy to reduce re-experience symptoms. Acta Psychiatr Scand 116: $145-153$.

Koch SB, van Zuiden M, Nawijn L, Frijling JL, Veltman DJ, Olff M (2016). Aberrant resting-state brain activity in posttraumatic stress disorder: a meta-analysis and systematic review. Depress Anxiety 33: 592-605.

Kuo JR, Kaloupek DG, Woodward SH (2012). Amygdala volume in combat-exposed veterans with and without posttraumatic stress disorder: a cross-sectional study. Arch Gen Psychiatry 69: 1080-1086.

Lanius RA, Bluhm R, Lanius U, Pain C (2006). A review of neuroimaging studies in PTSD: heterogeneity of response to symptom provocation. J Psychiatr Res 40: 709-729. 
Lanius RA, Williamson PC, Densmore M, Boksman K, Gupta MA, Neufeld RW et al (2001). Neural correlates of traumatic memories in posttraumatic stress disorder: a functional MRI investigation. Am J Psychiatry 158: 1920-1922.

Li L, Wu M, Liao Y, Ouyang L, Du M, Lei D et al (2014). Grey matter reduction associated with posttraumatic stress disorder and traumatic stress. Neurosci Biobehav Rev 43: 163-172.

Lyoo IK, Kim JE, Yoon SJ, Hwang J, Bae S, Kim DJ (2011). The neurobiological role of the dorsolateral prefrontal cortex in recovery from trauma. Longitudinal brain imaging study among survivors of the South Korean subway disaster. Arch Gen Psychiatry 68: 701-713.

Milad MR, Wright CI, Orr SP, Pitman RK, Quirk GJ, Rauch SL (2007). Recall of fear extinction in humans activates the ventromedial prefrontal cortex and hippocampus in concert. Biol Psychiatry 62: 446-454.

Morey RA, Gold AL, LaBar KS, Beall SK, Brown VM, Haswell CC et al (2012). Amygdala volume changes in posttraumatic stress disorder in a large case-controlled veterans group. Arch Gen Psychiatry 69: 1169-1178.

Ongur D, Price JL (2000). The organization of networks within the orbital and medial prefrontal cortex of rats, monkeys and humans. Cereb Cortex 10: 206-219.

Patel R, Spreng RN, Shin LM, Girard TA (2012). Neurocircuitry models of posttraumatic stress disorder and beyond: a metaanalysis of functional neuroimaging studies. Neurosci Biobehav Rev 36: 2130-2142.

Paulus MP, Stein MB (2006). An insular view of anxiety. Biol Psychiatry 60: 383-387.

Pitman RK, Rasmusson AM, Koenen KC, Shin LM, Orr SP, Gilbertson MW et al (2012). Biological studies of post-traumatic stress disorder. Nat Rev Neurosci 13: 769-787.

Quirk GJ, Beer JS (2006). Prefrontal involvement in the regulation of emotion: convergence of rat and human studies. Curr Opin Neurobiol 16: 723-727.

Rabinak CA, Angstadt M, Welsh RC, Kenndy AE, Lyubkin M, Martis B et al (2011). Altered amygdala resting-state functional connectivity in post-traumatic stress disorder. Front Psychiatry 2: 62 .

Saygin ZM, Osher DE, Augustinack J, Fischl B, Gabrieli JD (2011). Connectivity-based segmentation of human amygdala nuclei using probabilistic tractography. Neuroimage 56: 1353-1361.

Schiller D, Delgado MR (2010). Overlapping neural systems mediating extinction, reversal and regulation of fear. Trends Cogn Sci 14: 268-276.

Shin LM, Liberzon I (2010). The neurocircuitry of fear, stress, and anxiety disorders. Neuropsychopharmacoloy 35: 169-191.

Sripada RK, King AP, Garfinkel SN, Wang X, Sripada CS, Welsh RC et al (2012a). Altered resting-state amygdala functional connectivity in men with posttraumatic stress disorder. J Psychiatry Neurosci 37: 241-249.

Sripada RK, King AP, Welsh RC, Garfinkel SN, Wang X, Sripada CS et al (2012b). Neural dysregulation in posttraumatic stress disorder: evidence for disrupted equilibrium between salience and default mode brain networks. Psychosom Med 74: 904.

Tzourio-Mazoyer N, Landeau B, Papathanassiou D, Crivello F, Etard O, Delcroix N et al (2002). Automated anatomical labeling of activations in SPM using a macroscopic anatomical parcellation of the MNI MRI single-subject brain. Neuroimage 15: 273-289.

Westfall PH, Young SS (1993). Resampling-Based Multiple Testing: Examples and Methods for P-Value Adjustment. John Wiley \& Sons: New York, USA, Vol 279.

Woon FL, Hedges DW (2009). Amygdala volume in adults with posttraumatic stress disorder: a meta-analysis. J Neuropsychiatry Clin Neurosci 21: 5-12.

Yehuda R, LeDoux J (2007). Response variation following trauma: a translational neuroscience approach to understanding PTSD. Neuron 56: 19-32.

Yin Y, Jin C, Hu X, Duan L, Li Z, Song M et al (2011). Altered resting-state functional connectivity of thalamus in earthquakeinduced posttraumatic stress disorder: a functional magnetic resonance imaging study. Brain Res 1411: 98-107.

Supplementary Information accompanies the paper on the Neuropsychopharmacology website (http://www.nature.com/npp) 